\title{
Towards new background independent representations for Loop Quantum Gravity
}

\author{
Madhavan Varadarajan \\ Raman Research Institute, Bangalore 560080 \\ madhavan@rri.res.in
}

September 6, 2021

\begin{abstract}
Recently, uniqueness theorems were constructed for the representation used in Loop Quantum Gravity. We explore the existence of alternate representations by weakening the assumptions of the so called LOST uniqueness theorem. The weakened assumptions seem physically reasonable and retain the key requirement of explicit background independence. For simplicity, we restrict attention to the case of gauge group $U(1)$.
\end{abstract}

\section{Introduction}

Loop Quantum Gravity (LQG) is an attempt at canonical quantization of a reformulation of classical gravity in terms of a spatial $S U(2)$ connnection and its conjugate electric field. The representation underlying LQG is one in which the basic operators of the theory are holonomies of the connnection around spatial loops and electric fluxes through spatial surfaces. Much of the progress in LQG and many of its results (such as the discrete spectra of spatial geometric operators) depend on the detailed properties of this representation. Hence it is important to know if the choice of this representation is essentially unique, given appropriate physically reasonable requirements.

Work on this issue in recent years [1 has culminated in the formulation and proof of beautiful uniqueness theorems by Fleischhack [2] and Lewandowski, Okolow, Sahlmann and Thiemann (LOST) 3]. Here we turn our attention to the LOST theorem.

The two key inputs for the LOST theorem 1 are as follows:

(1) The algebra of holonomies and electric fluxes of which a representation is to be constructed.

\footnotetext{
${ }^{1}$ Please see the reference 3 for a detailed description of all the assumptions used in their proof.
} 
(2) The requirement that the representation contain a cyclic, spatially diffeomorphism invariant state.

While these are the simplest properties one may demand from a putative representation of a diffeomorphism invariant theory of $S U(2)$ connections and electric fields, one may enquire as to whether they are unduly restrictive. Let us discuss them one by one.

The holonomy- flux algebra of (1) agrees unambiguously with the corresponding Poisson bracket algebra except for the commutators of pairs of electric fluxes. While one may expect these to vanish in classical theory, the analysis of Reference [4] shows that there are subtelities related to the lack of sufficient (functional) differentiability of the holonomy- flux variables which belie this expectation. Motivated by this fact, LOST assume the commutators to take a particular form which is based on the interpretation of the flux operators as derivations on a space of cylindrical functions [3]. While these commutators, when evaluated in the representation used for LQG, have the physically appealing property of vanishing in a suitable "large scale", semiclassical approximation [4, they are based on structures which do not appear in the classical phase space. Hence, the replacement of these commutators by any other reasonable choice which vanishes in a suitable "large scale", semiclassical limit [4, would not be unnatural.

Further, note that the algebra involves only the holonomy- flux functions. This seems sufficient since any other function of interest can be built as (limits of) sums and products of these functions, and presumably, the corresponding operators may be constructed by the corresponding (limits of) sums and products of the holonomy and flux operators. However, in practice, in the representation currenty used in LQG, the generators of spatial diffeomorphisms cannot be constructed in this manner because the relevant limits do not exist in quantum theory. Indeed, only operators corresponding to finite spatial diffeomorphisms exist and these are defined through their natural action on the flux- holonomy operators rather than constructed from the latter. 2 Hence it is natural to consider for the quantum theory, a larger algebra generated by the holonomy, flux and finite spatial diffeomorphism operators. Clearly, we may only treat the finite spatial diffeomorphism operators as independent of the holonomy- flux ones provided that, in the chosen Hibert space representation, these operators cannot be constructed as suitable limits of combinations of the holonomy - flux operators. We shall return to this point in the concluding section of this paper.

Let us call the enlarged algebra $\mathcal{U D}$ (in contrast to the algebra $\mathcal{U}$ defined by LOST in [3]). The following relations hold on the generators of the algebra $\mathcal{U D}$ (these are, of course, in addition to the algebraic relations among

\footnotetext{
${ }^{2}$ The operators corresponding to finite spatial diffeomorphisms may also be constructed by their natural action on the space of generalised connections. However, this recipe too, does not derive from a construction of limits of flux-holonomy operators.
} 
elements of the algebra $\mathcal{U}):$

$$
\begin{aligned}
\hat{U}_{d_{1}} \hat{U}_{d_{2}} & =\hat{U}_{d_{1} \circ d_{2}}, \\
\hat{U}_{d} \hat{h}_{\alpha} \hat{U}_{d}^{\dagger} & =\hat{h}_{\alpha_{d}}, \\
\hat{U}_{d} \hat{E}_{\mathcal{S}, f} \hat{U}_{d}^{\dagger} & =\hat{E}_{\mathcal{S}_{d}, f_{d}}, \\
\hat{U}_{d}^{\dagger} & =\hat{U}_{d^{-1}}
\end{aligned}
$$

Here, $\hat{U}_{d}, \hat{h}_{\alpha}, \hat{E}_{\mathcal{S}, f}$ refer to the operators corresponding to the finite diffeomorphism $d$, the holonomy about a loop $\alpha$ and the flux through the surface $\mathcal{S}$ smeared by the Lie algebra valued test field $f$ which has support on $\mathcal{S}$; $\alpha_{d}, \mathcal{S}_{d}, f_{d}$ refer to the images of $\alpha, \mathcal{S}, f$ under the action of $d$. This completes our discussion of (1).

Next, consider the requirement (2). One consequence of (2) is that the representation is cyclic. Cyclicity, by itself, is a weak assumption since cyclicity is weaker than irreducibility and representations are usually classified in terms of their irreducible sectors. However, in light of the remarks in the previous paragraphs, it is not unnatural to consider cyclic representations of the enlarged algebra $\mathcal{U D}$ instead of the holonomy- flux algebra $\mathcal{U}$.

The further requirement that the representation contain a diffeomorphism invariant state on $\mathcal{U}$ which is also cyclic ensures that spatial diffeomorphisms act unitarily. It is quite natural to weaken this (rather strong) requirement to that of a unitary representation of spatial diffeomorphisms irrespective of the existence of a diffeomorphism invariant state, whether cyclic or not.

In this work we explore the consequences of these weakened requirements in the context of $U(1)$ connections and electric fields. We display a representation of $\mathcal{U D}$, inequivalent to the standard LQG type representation appropriate to gauge group $U(1)$, in which spatial diffeomorphisms act unitarily. The restriction to $U(1)$ is for simplicity. There seems to be no reason as to why our general ideas should not go through for the $S U(2)$ case relevant to LQG.

The layout of the paper is as follows. A detailed description of our constructions is presented in section 3. Since such an exposition may obscure the structures essential to our construction, we describe the broad underlying idea in section 2. This also serves to illustrate that there may exist implementations of our idea different from the particular one chosen in section 3. Section 3 also contains a discussion of open issues. Section 4 contains our concluding remarks. A key lemma is proved in the Appendix.

Depending on ones viewpoint on the validity of replacing $\mathcal{U}$ by $\mathcal{U D}$, the particular representation displayed here may be deemed to possess some undesirable features. We shall discuss this in section 3. However, as (at least to our knowledge) this is the first discussion of a representation different from the standard one which supports the holonomy- flux operators 
as well as a unitary action of spatial diffeomorphisms, at the very least our work may be viewed as initiating an exploration of background independent representations inequivalent to the standard one.

\section{A sketch of the underlying idea.}

Let $(\mathcal{O}, \mathcal{H})$ denote a representation of the $*$ - algebra $\mathcal{O}$ on the non- seperable Hilbert space $\mathcal{H}$. The idea is to construct a new representation of $\mathcal{O}$ in terms of 1 parameter families of states in $\mathcal{H}$. We use the following notation. $|\psi\rangle$ denotes an element of $\mathcal{H}$. A 1 parameter family of states in $\mathcal{H},|\psi(a)\rangle \in$ $\mathcal{H} \forall a \in R$ ( $R$ denotes the set of reals) is denoted by $\{|\psi(a)\rangle\}$. The inner product between $\left|\psi_{1}\right\rangle,\left|\psi_{2}\right\rangle \in \mathcal{H}$ is $\left\langle\psi_{1} \mid \psi_{2}\right\rangle$. Given a 1 parameter family of states $\{|\psi(a)\rangle\}$, the state at parameter value $a=a_{1}$ is $\left|\psi\left(a_{1}\right)\right\rangle$. Since $\left|\psi\left(a_{1}\right)\right\rangle$ belongs to the 1 parameter family of states $\{|\psi(a)\rangle\}$ we write $\left|\psi\left(a_{1}\right)\right\rangle \in$ $\{|\psi(a)\rangle\}$. Given a pair of 1 parameter families of states $\{|\psi(a)\rangle\},\{|\phi(b)\rangle\}$, we denote the inner product between $\left|\psi\left(a_{1}\right)\right\rangle \in\{|\psi(a)\rangle\}$ and $\left|\phi\left(b_{1}\right)\right\rangle \in\{|\phi(b)\rangle\}$ by $\left\langle\psi\left(a_{1}\right) \mid \phi\left(b_{1}\right)\right\rangle$.

We are interested in a certain set of 1 parameter families of states in $\mathcal{H}$. Call this set $\mathbf{B}$. Elements of $\mathbf{B}$ consist of 1 parameter families of states subject to the following restrictions:

(i) Let $\{|\psi(a)\rangle\} \in \mathbf{B}$. We require that $\left\langle\psi\left(a_{1}\right) \mid \psi\left(a_{2}\right)\right\rangle=\delta_{a_{1}, a_{2}}$ where $\delta_{a_{1}, a_{2}}$ is the Kronecker delta function 3 which equals unity when $a_{1}=a_{2}$ and vanishes otherwise. Note that the existence of such 1 parameter families presupposes that $\mathcal{H}$ is non-seperable.

(ii) Let $\{|\psi(a)\rangle\},\{|\phi(b)\rangle\} \in \mathbf{B}$. We require that for every value of $a$ there exists at most one value of $b$ such that $\langle\psi(a) \mid \phi(b)\rangle \neq 0$.

(iii) Clearly (ii) defines an invertible function $b(a)$ from (a subset of) $R$ into $R$. We require that this function be piecewise analytic i.e. the curve $(a, b(a))$ in the $a-b$ plane is required to be piecewise analytic.

(iv) We also require that the "overlap function" $f(a):=\langle\psi(a) \mid \phi(b(a))\rangle$ is piecewise analytic.

We shall use elements of $\mathbf{B}$ to define a basis for the new representation. Specifically, every basis element is in correspondence with a pair of labels, $(A,\{|\psi(a)\rangle\})$. Here $A$ denotes a piecewise smooth scalar of density $\frac{1}{2}$ on the real line and $\{|\psi(a)\rangle\} \in \mathbf{B}$. 4 Next, we define the inner product between

\footnotetext{
${ }^{3}$ Roughly speaking, we aim to construct a new representation in which the inner product is the Dirac delta function, $\delta\left(a_{1}, a_{2}\right)$ instead of the Kronecker delta function.

${ }^{4}$ More precisely (as we shall see) it is the equivalence class of $\{|\psi(a)\rangle\}$ under reparametrizations rather than $\{|\psi(a)\rangle\}$ itself which serves as an appropriate label.
} 
$(A,\{|\psi(a)\rangle\}),(B,\{|\phi(b)\rangle\})$ to be

$$
<A,\{|\psi(a)\rangle\} \mid B,\{|\phi(b)\rangle\}>=\int_{\mathcal{C}} d a\left|\frac{d b}{d a}\right|^{\frac{1}{2}} A^{*}(a) B(b(a))\langle\psi(a) \mid \phi(b(a))\rangle
$$

Here $\mathcal{C}$ denotes the piecewise analytic curve $(a, b(a))$ (see (iii)) above). It is straightforward to verify that the inner product (5) is hermitian and that the density weights of $A, B$ imply that equation (5) is independent of the specific parametrizations, $a$ and $b$, of $\{|\psi(a)\rangle\}$ and $\{|\phi(b)\rangle\}$. This suggests that we relabel our basis elements by half densities and equivalence classes of 1 parameter families of states under reparameterization. Accordingly, denote the equivalence class of 1 parameter families of states of which $\{|\psi(a)\rangle\}$ is a member, by $\Psi$, the set of basis states by $\mathcal{B}$ and elements of $\mathcal{B}$ by $|A, \Psi\rangle$. Then the inner product between $|A, \Psi\rangle$ and $|B, \Phi\rangle$ is given by the right hand side of (5) so that

$$
\langle A, \Psi \mid B, \Phi\rangle=\int_{\mathcal{C}} d a\left|\frac{d b}{d a}\right|^{\frac{1}{2}} A^{*}(a) B(b(a))\langle\psi(a) \mid \phi(b(a))\rangle
$$

Denote the new representation space by $V$. Any $|v\rangle \in V$ is a finite linear combination of elements of $\mathcal{B}$ so that $|v\rangle=\sum_{I=1}^{N} c_{I}\left|A_{I}, \Psi_{I}\right\rangle$ where $c_{I}$ are complex coefficients. We extend the scalar product (6) to all of $V$ by appropriate (anti-)linearity. Further, as suggested by equation ([6), we make the following identifications in $V$ :

$$
c|A, \Psi\rangle=|c A, \Psi\rangle
$$

for all complex $c$ and $|A, \Psi\rangle \in \mathcal{B}$. A final identification of vectors in $V$ is suggested by the linear structure of $\mathcal{H}$ and the scalar product (6) as follows.

Let $\left|A_{I_{i}}^{(i)}, \Psi_{I_{i}}^{(i)}\right\rangle,\left|B_{J_{j}}^{(j)}, \Phi_{J_{j}}^{(j)}\right\rangle \in \mathcal{B}, I_{i}=1, . ., L_{i}, J_{j}=1, . . M_{j}, i=1,2, j=$ 1,2 and let there exist the representatives $\left\{\left|\psi_{I_{i}}^{(i)}(a)\right\rangle\right\},\left\{\left|\phi_{J_{j}}^{(j)}(b)\right\rangle\right\}$ of $\Psi_{I_{i}}^{(i)}, \Phi_{J_{j}}^{j}$ in B. Further, let $\sum_{I_{1}=1}^{L_{1}} A_{I_{1}}^{(1)}(a)\left|\psi_{I_{1}}^{(1)}(a)\right\rangle=\sum_{I_{2}=1}^{L_{2}} A_{I_{2}}^{(2)}(a)\left|\psi_{I_{2}}^{(2)}(a)\right\rangle$ and $\sum_{J_{1}=1}^{M_{1}} B_{J_{1}}^{1}(b)\left|\phi_{J_{1}}^{(1)}(b)\right\rangle=\sum_{J_{2}=1}^{M_{2}} B_{J_{2}}^{(2)}(b)\left|\phi_{J_{2}}^{(2)}(b)\right\rangle$. Then, the identifications

$$
\sum_{I_{1}=1}^{L_{1}}\left|A_{I_{1}}^{(1)}, \Psi_{I_{1}}^{(1)}\right\rangle=\sum_{I_{2}=1}^{L_{2}}\left|A_{I_{2}}^{(2)}, \Psi_{I_{2}}^{(2)}\right\rangle
$$

and

$$
\sum_{J_{1}=1}^{M_{1}}\left|B_{J_{1}}^{(1)}, \Phi_{J_{1}}^{(1)}\right\rangle=\sum_{J_{2}=1}^{M_{2}}\left|B_{J_{2}}^{(2)}, \Phi_{J_{2}}^{(2)}\right\rangle
$$

are suggested by the fact that the expression

$$
c_{i j}:=\sum_{I_{i}=1}^{L_{i}}\left\langle A^{(i)}, \Psi_{I_{i}}^{(i)} \mid \sum_{J_{j}=1}^{N_{j}} B_{J_{j}}^{(j)}, \Psi_{J_{j}}^{(j)}\right\rangle
$$


is independent of $i, j$. This fact follows straightforwardly from the scalar product (6) and the piecewise analyticity properties described in (iii) and (iv). Denote by $V_{\text {new }}$ the vector space obtained after the identifications (7), (8) have been made in $V$. $V_{\text {new }}$ serves as the representation space for our new representation. Clearly, equation (6) provides a hermitian inner product on $V_{\text {new }}$. In the next section we shall show that the particular choice of 1 parameter families of states in $\mathcal{H}$ used for abelian gauge theory ensures that this inner product is also positive definite 5 and may be used to complete $V_{\text {new }}$ to a Hilbert space $\mathcal{H}_{\text {new }}$.

Finally, we turn to the representation of the $*$ - algebra $\mathcal{O}$ on $V_{\text {new }}$. The action of any $\hat{O} \in \mathcal{O}$ on $\mid A,\{|\psi(a)\rangle\}\rangle,\{|\psi(a)\rangle\} \in \mathbf{B}$, is defined to be $\mid A,\{\hat{O}|\psi(a)\rangle\}\rangle$. Here $\{\hat{O}|\psi(a)\rangle \in \mathcal{H} \forall a\}$ defines a new 1 parameter family of states. While this family need not satisfy (i)- (iv), we assume that it can be decomposed into a finite linear combination of 1 parameter families which do. Thus we assume that

$$
\hat{O}|\psi(a)\rangle=\sum_{I=1}^{N} A_{I}(a)\left|\psi_{I}(a)\right\rangle,
$$

where $\left\{\left|\psi_{I}(a)\right\rangle\right\} \in \mathbf{B}$ and $A_{I}$ are piecewise smooth scalar functions (of weight zero). We set $\left.\mid A,\{\hat{O}|\psi(a)\rangle\}\rangle=\sum_{I=1}^{N} \mid A A_{I},\left\{\left|\psi_{I}(a)\right\rangle\right\}\right\rangle$ so that

$$
\hat{O}|A, \Psi\rangle:=\sum_{I=1}^{N}\left|A A_{I}, \Psi_{I}\right\rangle
$$

in obvious notation. It follows from equation (8) that the definition (12) is independent of the particular decomposition of $\hat{O}|A, \Psi\rangle$ used and that it, in conjunction with the inner product (6) provides a $*$ - representation of the *- algebra $\mathcal{O}$ on $V_{\text {new }}$.

In the next section, we shall provide a precise implementation of these ideas for abelian gauge theory. Before we conclude this section, we mention a useful heuristic which serves to emphasize the point made in Footnote 3 , This is not required for the rest of the paper and the reader may proceed straight to section 2 if it so desires. Define $\left|\psi_{\text {new }}(a)\right\rangle,\left|\phi_{\text {new }}(b)\right\rangle$ through

$$
\begin{aligned}
|A, \Psi\rangle & =: \quad \int d a A(a)\left|\psi_{\text {new }}(a)\right\rangle \\
|B, \Phi\rangle & =: \quad \int d b B(b)\left|\phi_{\text {new }}(b)\right\rangle
\end{aligned}
$$

with the new inner product being

$$
\left.\left.\left\langle\psi_{\text {new }}(a) \mid \phi_{\text {new }}(b)\right\rangle>=\langle\psi(a)| \phi(b)\right)\right\rangle \delta(b, b(a))\left|\frac{d b}{d a}\right|^{\frac{1}{2}}
$$

\footnotetext{
${ }^{5}$ Specifically, in section 3 , the set $\mathbf{B}$ is such that any state $|v\rangle=\sum_{I=1}^{N} c_{I}\left|A_{I}, \Psi_{I}\right\rangle$ can be rewritten using the equations (7) and (8) as $|v\rangle=\sum_{I=1}^{M} d_{J}\left|B_{J}, \Phi_{J}\right\rangle$ with $\left\langle B_{J}, \Phi_{J} \mid B_{K}, \Phi_{K}\right\rangle=0$ for $J \neq K$. Postive definiteness then follows from equation (6).
} 
In this notation we have

$$
\langle A, \Psi|=: \int d a A^{*}(a)\left\langle\psi_{\text {new }}(a)\right|
$$

so that

$$
\langle A, \Psi \mid B, \Phi\rangle:=\int d a d b A^{*}(a) B(b)\left\langle\psi_{\text {new }}(a) \mid \phi_{\text {new }}(b)\right\rangle>
$$

which reduces to equation (6) if we use the definition (15). This notation brings out the Dirac delta normalization mentioned in Footnote 3 and easily lends itself to the linearity based identifications (7) and (8).

\section{The new representation for the case of gauge group $U(1)$.}

We set $\mathcal{O}$ to be the $*$ - algebra $\mathcal{U D}$ (see the discussion associated with the equations (11)- (4)) and $(\mathcal{O}, \mathcal{H})$ to be the standard "flux network" representation [5, 6] which is the abelian analog of the spin network representation currently used in LQG.

We provide a brief review of the flux network representation in section 3.1. In section 3.2, we define the set $\mathbf{B}$ of 1 parameter families of states on which the new representation is based and show that the inner product (6) is positive definite on the representation space $V_{\text {new }}$. In section 3.3 , we display the action of the basic operators $\hat{h}_{\alpha}, \hat{E}(f), \hat{U}_{d}$ and show that the assumption (11) is valid. As mentioned in section 2 , this ensures that $\mathcal{U D}$ is represented on $\mathcal{H}_{\text {new }}$.

\subsection{Review of the $U(1)$ flux network representation.}

This section provides a brief review of the flux network representation for a difffeomorphism invariant theory of $U(1)$ connections and conjugate electric fields. Our primary aim is to establish notation. We refer the reader to the review article [7] and the references contained therein for a complete presentation.

Let $\Sigma$ be a 3 dimensional, compact, real analytic manifold without boundary. The phase space variables are a $U(1)$ connection $A_{a}(x)$ and its conjugate (unit density weight) electric field $E^{a}(x)$. Let $\alpha$ be a closed oriented graph composed of closed analytic edges $e_{J}, J=1, . ., M$. Let each edge $e_{J}$ be labelled by an integer $p_{J}$ such that at each vertex the sum of integers labelling outgoing edges equals that for incoming edges. The holonomy of the connection associated with this labelled graph is $h_{\alpha, \vec{p}}=$ $\exp \left(i \sum_{J=1}^{M} \int_{e_{J}} A_{a} d x^{a}\right)$. As shown in Reference [5] these graph holonomies are in correspondence with the more commonly used loop holonomies i.e. 
for every such labelled graph $\alpha, \vec{p}$ there exists a loop $\beta$ such that $h_{\alpha, \vec{p}}=h_{\beta}$ where $h_{\beta}:=\exp \left(i \oint_{\beta} A_{a} d x^{a}\right)$ is the usual loop holonomy.

The smeared electric flux through a 2 dimensional surface $\mathcal{S}$ is $E_{\mathcal{S}, f}=$ $\int_{\mathcal{S}} f(x) E^{a}(x) d^{2} s_{a}$. Here $f(x)$ is a smooth function of compact support on $\mathcal{S}$. The surface $\mathcal{S}$ is chosen as in Reference [4. For our purposes it is important to note that this choice is such that the surface is semianalytic (Please see Reference [3] for a defintion of semianalyticity.). The holonomy- flux Poisson bracket algebra leads unambiguously to the following commutators in quantum theory:

$$
\left[\hat{h}_{\alpha_{1}, \vec{p}_{1}}, \hat{h}_{\alpha_{2}, \vec{p}_{2}}\right]=0, \quad\left[\hat{h}_{\alpha, \vec{p}}, \hat{E}_{\mathcal{S}, f}\right]=-\hbar\left(\sum_{i} f\left(x_{i}\right) \sum_{I_{i}} p_{I_{i}} \kappa_{I_{i}}\right) \hat{h}_{\alpha, \vec{p}},
$$

where $i$ labels the transverse intersections of $\alpha$ with $\mathcal{S}$ (by which we mean that at least one edge of $\alpha$ is transverse to $\mathcal{S}$ at the intersection point), $I_{i}$ ranges over the edges of $\alpha$ which intersect $\mathcal{S}$ transeversely at the point $x_{i}$ and $\kappa_{I_{i}}=1$ or -1 depending on the relative orientations and positions of $e_{I_{i}}$ and $\mathcal{S}$ (it turns out that without loss of generality one can always arrange for $x_{i}$ to be a vertex of $\alpha$; see Footnote 6 and References [8, 5]). Finally, the commutator of a pair of fluxes is

$$
\left[\hat{E}_{\mathcal{S}_{1}, f_{1}}, \hat{E}_{\mathcal{S}_{2}, f_{2}}\right]=0 .
$$

We emphasize here that in the case of $U(1)$ gauge group, the commutator of two electric flux variables (19) vanishes unambiguously [4] and the subtlety mentioned in the Introduction does not arise. The holonomy and flux operators together with their commutators (18)- (19) generate the algebra $\mathcal{U}$ on which the $*$ - relations are induced from the adjointness properties of the holonomy- flux operators:

$$
\left(\hat{h}_{\alpha, \vec{p}}\right)^{\dagger}=\left(\hat{h}_{\alpha, \vec{p}}\right)^{-1} \quad\left(\hat{E}_{\mathcal{S}, f}\right)^{\dagger}=\hat{E}_{\mathcal{S}, f} .
$$

The $*$ - algebra $\mathcal{U}$ is represented on the Hilbert space $\mathcal{H}$ which is spanned by an orthonormal basis of flux network states. For simplicity, we shall restrict attention to gauge invariant states. Each such state (with the exception of the state $|0\rangle$ defined below) is in correspondence with a closed oriented graph, every edge of which is labelled by a (non- trivial) representation of the Lie algebra of $U(1)$ i.e. by a non- zero integer. Each edge is required to be analytic and closed and at each vertex the sum of the integer labels of the incoming edges equals that of the outgoing edges. Consider such a graph $\gamma$ with edges $e_{I}, I=1, . ., N$, each labelled by a non-zero integer $n_{I}$. The corresponding flux network state is denoted by $|\gamma, \vec{n}\rangle$. The flux network state associated with the trivial graph (with no edges) is denoted by $|0\rangle$.

The action of the holonomy operator, $\hat{h}_{\alpha, \vec{p}}$, on the flux network state $|\gamma, \vec{n}\rangle$ is

$$
\hat{h}_{\alpha, \vec{p}}|\gamma, \vec{n}\rangle=|\gamma \cup \alpha, \vec{n} \cup \vec{p}\rangle
$$


The labels on the right hand side are defined as follows. Consider any (closed, oriented) graph (with closed analytic edges) finer than $\gamma$ and $\alpha$. 6 Any edge $e$ of this graph is labelled as follows (below $e_{I}$ denotes the $I$ th edge of $\gamma$ and $e_{J}^{\alpha}$ the Jth edge of $\alpha$ ):

(a) If $e \subset e_{I}, e \subset e_{J}^{\alpha}$ and $e, e_{I}, e_{J}^{\alpha}$ have the same orientation, $e$ is assigned the label $n_{I}+p_{J}$. In case of orientation mismatches with only $e_{I}$, only $e_{J}^{\alpha}$ or both $e_{I}$ and $e_{J}^{\alpha}$ the label is $-n_{I}+p_{J}, n_{I}-p_{J}$ or $-n_{I}-p_{J}$.

(b) If $e \subset e_{I}$ and $e$ intersects $\alpha$ at most at isolated points, $e$ is assigned the label $n_{I}$ if its orientation is the same as that induced from $e_{I}$ else it is labelled $-n_{I}$.

(c) If $e \subset e_{J}^{\alpha}$ and $e$ intersects $\gamma$ at most at isolated points, then $e$ is assigned the label $p_{J}$ if its orientation is the same as that of $e_{J}^{\alpha}$ (else it is labelled $\left.-p_{J}\right)$.

The pair $\gamma \cup \alpha, \vec{n} \cup \vec{p}$ is defined by (a)- (c) above with the additional caveat that any edge $e$ whose integer label vanishes as a result of (a) is dropped from the graph.

The action of the electric flux operator $\hat{E}_{\mathcal{S}, f}$ on the state $|\gamma, \vec{n}\rangle$ is

$$
\hat{E}_{\mathcal{S}, f}|\gamma, \vec{n}\rangle=\hbar\left(\sum_{i} f\left(x_{i}\right) \sum_{I_{i}} n_{I_{i}} \kappa_{I_{i}}\right)|\gamma, \vec{n}\rangle,
$$

where $x_{i}, I_{i}, \kappa_{I_{i}}$ are defined as in equation (18). The operator $\hat{U}_{d}$ which corresponds to the action of the finite diffeomorphism $d$ (see equations (1)(44)) acts on the state $|\gamma, \vec{n}\rangle$ as

$$
\hat{U}_{d}|\gamma, \vec{n}\rangle=\left|\gamma_{d}, \vec{n}\right\rangle
$$

where on the right hand side, $\gamma_{d}$ is the image of the graph $\gamma$ under the diffeomorphism $d$ and the image of the edge $e_{I}$ of the graph $\gamma$ under $d$ is labelled by $n_{I}$. We shall restrict attention to analytic diffeomorphisms $d$, so that $\gamma_{d}$ is also a piecwise analytic graph.

In addition to their action on $|\gamma, \vec{n}\rangle$ (21)- (23), the above operators act as follows on the trivial graph state.

$$
\hat{h}_{\alpha, \vec{p}}|\circ\rangle=|\alpha, \vec{p}\rangle,
$$

We also have

$$
\hat{E}_{\mathcal{S}, f}|\circ\rangle=0
$$

\footnotetext{
${ }^{6} \mathrm{~A}$ graph $\gamma_{1}$ is said to be finer than a graph $\gamma_{2}$ iff every edge of the latter can be composed of edges in the former. We may also refer to $\gamma_{2}$ as being coarser than $\gamma_{1}$. A flux network state, strictly speaking, is labelled by an equivalence class of graphs and integer labellings where the pair $\gamma_{1}, \vec{n}_{1}$ and the pair $\gamma_{2}, \vec{n}_{2}$ are equivalent if the images of $\gamma_{1}$ and $\gamma_{2}$ in $\Sigma$ are identical and either (a) $\gamma_{1}$ is finer than $\gamma_{2}$ and the edges in the former which compose to yield an edge in the latter are all labelled by the (same) integer which labels the edge in the latter or (b) vice versa. We also note that flipping the orientation of edges in a flux network state is the same as retaining the orientation and flipping the sign of the integer labels.
} 
and

$$
\hat{U}_{d}|0\rangle=|0\rangle,
$$

It can be verified that the equations (21)- (26) together with the orthonormality of the flux network basis, provide a $*$ - representation for the * - algebra $\mathcal{U D}(11)-(4)$ with $\mathcal{U}$ defined through (18)- (19) and (20).

\subsection{Construction of the new Hilbert space.}

We define the set $\mathbf{B}$ of 1 parameter families of states as follows. Let $\gamma$ be a closed graph with closed analytic edges $e_{I}, I=1, . ., N$. Let $\xi$ be a real analytic vector field on $\Sigma$ and $U$ an open neighbourhood in $\Sigma$. Let $\phi_{\xi}(s), s \in R$, denote the one parameter family of diffeomorphisms of $\Sigma$ generated by $\xi$ with $\phi_{\xi}(0)$ being the identity map. Let $\gamma(\xi, s)$ be the graph obtained by the action of $\phi_{\xi}(s)$ on $\gamma$ i.e. $\gamma(\xi, s)=\phi_{\xi}(s) \gamma$. We subject $\gamma, \xi, U$ to the following restrictions:

(a) We require that each $e_{I}$ admits a non-self intersecting, open, analytic extension $\tilde{e}_{I}$ such that $\tilde{e}_{I} \subset U$.

(b) We require that $\xi$ be nonvanishing in $U$ and transverse to every $\tilde{e}_{I}, I=$ $1, . ., N$.

Clearly, there exists an open neighbourhood $S$ of the origin such that for every $s, s^{\prime}, s^{\prime \prime} \in S$,

$$
\begin{gathered}
\phi_{\xi}(s) \tilde{e}_{I} \subset U, \quad I=1, . ., N, \\
\gamma\left(\xi, s^{\prime}\right)=\gamma\left(\xi, s^{\prime \prime}\right) \quad \text { iff } s^{\prime}=s^{\prime \prime} .
\end{gathered}
$$

Consider a flux network $|\gamma, \vec{n}\rangle$ based on the graph $\gamma$. Let $|\gamma(\xi, s), \vec{n}\rangle$ denote the flux network based on $\gamma(\xi, s)$ such that the $I$ th edge of $\gamma(\xi, s)$, namely $\phi_{\xi}(s) e_{I}$, is labelled by $n_{I}$. Finally, let $\mathbf{S} \subset S$ be a closed interval containing the origin. Then the set $\mathbf{B}$ consists of the 1 parameter families of states $\left\{\hat{h}_{\alpha, \vec{p}}|\gamma(\xi, s), \vec{n}\rangle, s \in \mathbf{S}\right\}$ for all possible choices of $\alpha, \vec{p}, \gamma, \vec{n}, \xi, \mathbf{S}$. If $\alpha$ is chosen to be the trivial graph, o, there is no labelling $\vec{p}$ and the 1 parameter family of states is just $\{|\gamma(\xi, s), \vec{n}\rangle, s \in \mathbf{S}\}$.

Clearly, equation (28), in conjunction with the orthogonality of flux network states based on different graphs, ensures that (i),(ii) of section 2 hold. We now show that (iii) also holds. Let $\left\{\hat{h}_{\alpha_{i}, \vec{p}_{i}}\left|\gamma_{i}\left(\xi_{i}, s_{i}\right), \vec{n}_{i}\right\rangle, s_{i} \in \mathbf{S}_{i}\right\}, i=$ 1,2 be a pair of states such that infinitely many points $\left(s_{1}, s_{2}\left(s_{1}\right)\right)$ exist where the states are non- orthogonal (here $\alpha_{i}$ could also be the trivial graph in which case the labelling $\vec{p}_{i}$ is absent). Specifically, let $s_{1 \min }$ and $s_{1 \max }$ be the minimum and maximum values of $s_{1}$ for which $s_{2}\left(s_{1}\right)$ exists. Then there are infinitely many points $s_{1}, s_{1} \in\left[s_{1 \text { min }}, s_{1 \max }\right]$ such that $s_{2}\left(s_{1}\right)$ exists. Next, note that the images of of $\gamma_{1}\left(\xi_{1}, s_{1}\right), \gamma_{2}\left(\xi_{2}, s_{2}\left(s_{1}\right)\right)$ agree in $\Sigma$. Hence, we choose the two graphs to have the same number of edges (That this does not entail any loss of generality follows from Footnote 6.). Let the Ith edge of $\gamma\left(\xi_{1}, s_{1}\right)$ (i.e. $\left.\phi_{\xi_{1}}\left(s_{1}\right) e_{1 I}\right)$ agree with the $J$ th edge of $\gamma\left(\xi_{2}, s_{2}\left(s_{1}\right)\right.$ ) (i.e. $\left.\phi_{\xi_{2}}\left(s_{2}\left(s_{1}\right)\right) e_{2 J}\right)$. While in principle $J$ could be a function of both $I$ and $s_{1}$, 
the fact that $\gamma_{1}, \gamma_{2}$ have a finite number of edges ensures that for infinitely many points $\left(s_{1}, s_{2}\left(s_{1}\right)\right)$ there exists $J=J(I), I=1, . ., N$ independent of $s_{1}$. An application of Lemma 1 of the Appendix to the edges $\tilde{e}_{1 I}, \tilde{e}_{2 J(I)}$ shows that the surfaces $\mathcal{S}_{1 I}, \mathcal{S}_{2 J}$ generated by the action of $\phi_{\xi_{1}}\left(s_{1}\right), \phi_{\xi_{2}}\left(s_{2}\right)$ on the edges $e_{1 I}, e_{2 J(I)}$ are analytic with analytic charts $\left(t_{1 I}, s_{1}\right),\left(t_{2 J(I)}, s_{2}\right)$ where $t_{1 I}, t_{2 J(I)}$ are analytic parameterizations of $\tilde{e}_{1 I}, \tilde{e}_{2 J(I)}$. Since these surfaces intersect at infinitely many curves, the portion of $\mathcal{S}_{1 I}$ between the curves $s_{1}=s_{1 \min }$ and $s_{1}=s_{1 \max }$ must coincide with the portion of $\mathcal{S}_{2 J(I)}$ between the curves $s_{2}\left(s_{1 \min }\right)$ and $s_{2}\left(s_{1 \max }\right)$. Then it follows from the analyticity of the charts mentioned above that $s_{2}\left(s_{1}\right)$ is an analytic function and hence that (iii) of section 2 holds. 7

Finally, it follows from the orthonormality of the flux network states that the relevant "overlap function" of (iv) is piecewise constant and hence piecewise analytic.

The next step is to define the set $\mathcal{B}$. In what follows, we will drop the label $\xi$ from the set of labels characterising states in $\mathbf{B}$ and it will be understood that any 1 parameter family of graphs, $\gamma(s)$, has been obtained by dragging the graph $\gamma$ along the orbits of some analytic vector field in the manner discussed above. As in section 2, basis elements for our new representation are in correspondence with an element of $\mathbf{B}$ together with a piecewise smooth scalar density of weight $\frac{1}{2}$. We designate such elements by $|C, \alpha, \vec{p},\{\gamma(s), \vec{n}, s \in \mathbf{S}\}\rangle$ (and by $|C,\{\gamma(s), \vec{n}, s \in \mathbf{S}\}\rangle$ for the case $\alpha=$ o), where $C$ is a scalar half density of compact support such that $C(s)$ is supported in $\mathbf{S}$. As in section 2, two such elements are to be identified if they are related by analytic reparametrisations. Specifically, the set $(C, \gamma(s), s \in$ $\mathbf{S})$ is equivalent to the set $\left(C, \gamma^{\prime}\left(s^{\prime}\right), s^{\prime} \in \mathbf{S}^{\prime}\right)$ if $s\left(s^{\prime}\right)$ is an analytic bijection from $\mathbf{S}^{\prime}$ to $\mathbf{S}$ such that

$$
\gamma^{\prime}\left(s^{\prime}\right)=\gamma\left(s\left(s^{\prime}\right)\right), \quad \forall s^{\prime} \in \mathbf{S}^{\prime} .
$$

In addition, since $C$ is a half density, we have the

$$
C\left(s^{\prime}\right)=C\left(s\left(s^{\prime}\right)\right)\left|d s / d s^{\prime}\right|^{\frac{1}{2}}
$$

with $C\left(s^{\prime}\right)$ being supported in $\mathbf{S}^{\prime}$. We refer to the equivalence class of which $|C, \alpha, \vec{p},\{\gamma(s), \vec{n}, s \in \mathbf{S}\}\rangle$ is a member 8 by $|C, \Gamma, \alpha, \vec{p}, \vec{n}\rangle$ and that of $|C,\{\gamma(s), \vec{n}, s \in \mathbf{S}\}\rangle$ by $|C, \Gamma, \vec{n}\rangle$. Here, the information in $\mathbf{S}$ is implicit

\footnotetext{
${ }^{7}$ It is not difficult to see (for example via a straightforward application of the CauchyKowalewski theorem [9] to a 3 dimensional chart obtained by dragging a 2 dimensional patch along the orbits of a suitably defined analytic vector field) that the fact that $\left(t_{1 I}, s_{1}\right)$ and $\left(t_{2 J(I)}, s_{2}\right)$ define analytic charts on a common analytic surface implies that the latter are analytic functions of the former. In particular, $s_{2}$ is an analytic function of $t_{1 I}$ and $s_{1}$; however the fact that the analytic function $s_{2}$ is independent of $t_{1 I}$ for infinitely many values of $s_{1}$ implies that $s_{2}$ is an analytic function only of $s_{1}$.

${ }^{8}$ Strictly speaking this equivalence class should contain only elements of $\mathbf{B}$ which means that only those analytic reparameterizations should be permitted for which the parameter
} 
in the support of the half density $C$. Thus, the set $\mathcal{B}$ is composed of elements of the form $|C, \alpha, \vec{p}, \Gamma, \vec{n}\rangle,|C, \Gamma, \vec{n}\rangle$. The inner product (6) between $\left|C_{1}, \alpha_{1}, \vec{p}_{1}, \Gamma_{1}, \vec{n}_{1}\right\rangle$, and $\left|C_{2}, \alpha_{2}, \vec{p}_{2}, \Gamma_{2}, \vec{n}_{2}\right\rangle$, is

$$
\begin{gathered}
\left\langle C_{1}, \alpha_{1}, \vec{p}_{1}, \Gamma_{1}, \vec{n}_{1} \mid C_{2}, \alpha_{2}, \vec{p}_{2}, \Gamma_{2}, \vec{n}_{2}\right\rangle= \\
\int_{\mathcal{C}} d s_{1}\left|\frac{d s_{2}}{d s_{1}}\right|^{\frac{1}{2}} C_{1}^{*}\left(s_{1}\right) C_{2}\left(s_{2}\left(s_{1}\right)\right)\left\langle\gamma_{1}\left(s_{1}\right) \cup \alpha_{1}, \vec{n}_{1} \cup \vec{p}_{1} \mid \gamma\left(s_{2}\left(s_{1}\right)\right) \cup \alpha_{2}, \vec{n}_{2} \cup \vec{p}_{2}\right\rangle
\end{gathered}
$$

Clearly, on the curve $\mathcal{C}$ it must be the case that the flux networks $\mid \gamma_{1}\left(s_{1}\right) \cup$ $\left.\alpha_{1}, \vec{n}_{1} \cup \vec{p}_{1}\right\rangle$ and $\left|\gamma\left(s_{2}\left(s_{1}\right)\right) \cup \alpha_{2}, \vec{n}_{2} \cup \vec{p}_{2}\right\rangle$ are identical so that the equation (31) reduces to

$$
\left\langle C_{1}, \alpha_{1}, \vec{p}_{1}, \Gamma_{1}, \vec{n}_{1} \mid C_{2}, \alpha_{2}, \vec{p}_{2}, \Gamma_{2}, \vec{n}_{2}\right\rangle=\int_{\mathcal{C}} d s_{1}\left|\frac{d s_{2}}{d s_{1}}\right|^{\frac{1}{2}} C_{1}^{*}\left(s_{1}\right) C_{2}\left(s_{2}\left(s_{1}\right)\right) .
$$

It is also straightforward to verify that the same equation holds with the left hand side replaced by $\left\langle C_{1}, \Gamma_{1}, \vec{n}_{1} \mid C_{2}, \Gamma_{2}, \vec{n}_{2}\right\rangle$. The next step is use the elements of $\mathcal{B}$ to generate the vector space $V_{\text {new }}$ subject to the identifications (7) and (8). The inner product (31) is extended to $V_{\text {new }}$ by appropriate linearity and antilinearity. The general considerations of section 2 show that this inner product is Hermitian on $V_{\text {new }}$. We now demonstrate that it is also positive definite.

We present the argument for positive definiteness in a form readily generalizable to the non- abelian case. Our strategy (along the lines of Footnote 51) is to rewrite any linear combination of elements belonging to $\mathcal{B}$ as one in which each term is based on a 1 parameter family of graphs such that for any pair of terms, the corresponding pair of 1 parameter families of graphs have no common images in $\Sigma$. Let $|v\rangle=\sum_{i=1}^{m}\left|\Psi_{i}\right\rangle \in V_{\text {new }}$ where

$$
\left|\Psi_{i}\right\rangle=\left|C_{i}, \alpha_{i}, \vec{p}_{i}, \Gamma_{i}, \vec{n}_{i}\right\rangle \in \mathcal{B}
$$

Let $j$ be such that $\left\langle\Psi_{1} \mid \Psi_{j}\right\rangle \neq 0$ so that $\gamma_{j}\left(s_{j}\right)$ agrees with $\gamma_{1}\left(s_{1}\right)$ (almost) everywhere along the curve $\mathcal{C}_{1 j}$ in the $s_{1}-s_{j}$ plane. Using equation (8) and an appropriate analytic reparametrization, we have that

$$
\left|C_{j}, \alpha_{j}, \vec{p}_{j}, \Gamma_{j}, \vec{n}_{j}\right\rangle:=\left|C_{1 j}, \alpha_{j}, \vec{p}_{j}, \Gamma_{1}, \vec{n}_{j}\right\rangle+\left|C_{j}^{\prime}, \alpha_{j}, \vec{p}_{j}, \Gamma_{j}, \vec{n}_{j}\right\rangle,
$$

where

$$
\begin{aligned}
C_{1 j}\left(s_{1}\right) & =C_{j}\left(s_{j}\left(s_{1}\right)\right)\left|\frac{d s_{j}}{d s_{1}}\right|^{\frac{1}{2}} \forall s_{1} \text { such that } s_{j}\left(s_{1}\right) \text { exists, } \\
& =0 \text { elsewhere, } \\
C_{j}^{\prime}\left(s_{j}\right) & =0 \forall s_{j} \text { such that }\left(s_{1}, s_{j}\right) \in \mathcal{C}_{1 j} \\
& =C_{j}\left(s_{j}\right) \text { elsewhere. }
\end{aligned}
$$

takes values in a closed set containing the origin. However, we find it convenient to admit all possible analytic reparametrisations. Hence given an element of $\mathbf{B}$, its equivalence class contains all 1 parameter families which are related to it via arbitrary analytic reparameterizations. Thus, if $\mathbf{S}$ in the discussion above contains the origin, it is not necessary that $\mathbf{S}^{\prime}$ does. 
Next, define, for $i \neq 1$,

$$
\begin{aligned}
\left|\Psi_{i}^{\prime}\right\rangle & =\left|C_{i}^{\prime}, \alpha_{i}, \vec{p}_{i}, \Gamma_{i}, \vec{n}_{i}\right\rangle \text { if } \mathcal{C}_{1 i} \text { exists } \\
& =\left|C_{i}, \alpha_{i}, \vec{p}_{i}, \Gamma_{i}, \vec{n}_{i}\right\rangle \text { otherwise }
\end{aligned}
$$

and

$$
\left.\left|\Psi_{1}^{\prime}>=\right| C_{1}+\sum_{j} C_{1 j}, \alpha_{1}, \vec{p}_{1}, \Gamma_{1}, \vec{n}_{1}\right\rangle,
$$

where the sum is over all $j$ such that the curve $\mathcal{C}_{1 j}$ exists. Then using equation (8), we have that $|v\rangle=\sum_{i=1}^{m}\left|\Psi_{i}^{\prime}\right\rangle$ where $\left\langle\Psi_{1}^{\prime} \mid \Psi_{i}^{\prime}\right\rangle=0$ for all $i \neq 1$. Next, apply this procedure to the sum $\sum_{i=2}^{m}\left|\Psi_{i}^{\prime}\right\rangle$ so as to "orthogonalise" with respect to $\left|\Psi_{2}^{\prime}\right\rangle$. Clearly, by repeating this procedure enough times, we may rewrite $|v\rangle$ in the form

$$
|v\rangle=\sum_{i=1}^{m}\left|D_{i}, \alpha_{i}, \vec{p}_{i}, \Gamma_{i}, \vec{n}_{i}\right\rangle
$$

such that $\left\langle D_{i}, \alpha_{i}, \vec{p}_{i}, \Gamma_{i}, \vec{n}_{i} \mid D_{j}, \alpha_{j}, \vec{p}_{j}, \Gamma_{j}, \vec{n}_{j}\right\rangle=0$ for $i \neq j$. It is then straightforward to see that $\langle v \mid v\rangle$ may be evaluated using equations (31) and (43) to yield the manifestly positive definite expression:

$$
\langle v \mid v\rangle=\sum_{i=1}^{m} \int d s_{i}\left|D_{i}\left(s_{i}\right)\right|^{2} .
$$

The same argument goes through even if some or all of the $\alpha_{i}$ correspond to the trivial graph.

The inner product can be used to complete $V_{\text {new }}$ to the Hilbert space $\mathcal{H}_{\text {new }}$.

\subsection{Representation of operators on the Hilbert space.}

In this section we demonstrate the validity of the assumption (11) in the context of the new representation for abelian gauge fields. We define the action of the operators $\hat{h}_{\alpha, \vec{p}}, \hat{E}_{\mathcal{S}, f}$ and $\hat{U}_{d}$ in accordance with section 2. The holonomy operator acts as follows.

$$
\begin{gathered}
\hat{h}_{\alpha, \vec{p}}|C, \beta, \vec{q}, \Gamma, \vec{n}\rangle=|C, \beta \cup \alpha, \vec{q} \cup \vec{p}, \Gamma, \vec{n}\rangle, \\
\hat{h}_{\alpha, \vec{p}}|C, \Gamma, \vec{n}\rangle=|C, \alpha, \vec{p}, \Gamma, \vec{n}\rangle .
\end{gathered}
$$

Clearly this action satisfies the assumption (11). The flux operator acts as follows.

$$
\begin{gathered}
\hat{E}_{\mathcal{S}, f}|C, \alpha, \vec{p}, \Gamma, \vec{n}\rangle=\hbar\left|C C_{\alpha, \vec{p}, \Gamma, \vec{n}}^{\mathcal{S}, f}, \alpha, \vec{p}, \Gamma, \vec{n}\right\rangle \\
\hat{E}_{\mathcal{S}, f}|C, \Gamma, \vec{n}\rangle=\hbar\left|C C_{\Gamma, \vec{n}}^{\mathcal{S}, f}, \Gamma, \vec{n}\right\rangle
\end{gathered}
$$


where

$$
\begin{gathered}
C_{\alpha, \vec{p}, \Gamma, \vec{n}}^{\mathcal{S}, f}(s)=C_{\Gamma, \vec{n}}^{\mathcal{S}, f}(s)+\left(\sum_{i_{\alpha}} f\left(x_{i_{\alpha}}\right) \sum_{I_{i_{\alpha}}} p_{I_{i_{\alpha}}} \kappa_{I_{i_{\alpha}}}\right), \\
C_{\Gamma, \vec{n}}^{\mathcal{S}, f}(s)=\left(\sum_{i_{s}} f\left(x_{i_{s}}\right) \sum_{I_{i_{s}}} n_{I_{i_{s}}} \kappa_{I_{i_{s}}}\right) .
\end{gathered}
$$

The notation is similar to that in equation (18) and the subscripts $\alpha, s$ are used to designate indices appropriate to the graphs $\alpha, \gamma(s)$ and their intersections with $\mathcal{S}$. Thus, for example, $i_{s}$ ranges over the (transverse) intersections of $\gamma(s)$ with $\mathcal{S}$ and $I_{i_{s}}$ over the (transverse) edges of $\gamma(s)$ ending/begining at the interesction point $x_{i_{s}}$. If $C_{\alpha, \vec{p}, \Gamma, \vec{n}}^{\mathcal{S}, f}, C_{\Gamma, \vec{n}}^{\mathcal{S}, f}$ are piecewise smooth, then the right hand sides of the above equations are in $\mathcal{B}$. Hence we need to show that $C_{\Gamma, \vec{n}}^{\mathcal{S}, f}$ is a piecewise smooth function.

Note that the surface $\mathcal{S}$ can intersect the graph $\gamma(s)$ at most a finite number of times. Denote the $I$ th edge of $\gamma(s)$ by $e_{I}(s)$ and denote the surface traced out by $e_{I}(s)$ as $s$ varies, by $\mathcal{S}_{I}$. Lemma 1 of the Appendix implies that $\mathcal{S}_{I}$ is an analytic surface with piecewise analytic boundary. Note that both $\mathcal{S}$ and $\mathcal{S}_{I}$ are semianalytic surfaces (see Reference [3] and the references therein for a definition of semianalyticity). It follows from Reference [3] that $\mathcal{S} \cap \mathcal{S}_{I}$ is the union of a finite number of (a) isolated points, (b) piecewise analytic curves, and (c) 2 dimensional semianalytic surfaces. Isolated points are of measure zero in $s$ space and can therefore be ignored. The contributions from (c) to $C_{\Gamma, \vec{n}}^{\mathcal{S}, f}$ vanish since the associated edges are tangential to $\mathcal{S}$. Consider an analytic segement $\tau$ of (b) with analytic parameterization $u$ so that $\tau$ traces out the curve $\left(t_{I}(u), s(u)\right)$ on $\mathcal{S}_{\mathcal{I}}$ where $t_{I}$ is the parameter along the edge $e_{I}$. By Lemma $1, s(u)$ is an analytic function. Hence either

(1) $\frac{d s}{d u}=0$ at a finite number of points, or,

(2) $\tau$ is along some edge $e_{I}(s)$.

Case (2) is again one of tangential intersection and does not contribute to $C_{\Gamma, \vec{n}}^{\mathcal{S}, f}$. Since the only nontrivial contributions are from Case (1) and since the number of edges of $\gamma(s)$ is finite (and independent of $s$ ), it is straightforward to see that $C_{\Gamma, \vec{n}}^{\mathcal{S}, f}$ is a bounded, piecewise constant function of $s$.

Finally the operator $\hat{U}_{d}$ acts as follows:

$$
\begin{gathered}
\hat{U}_{d}|C, \beta, \vec{q}, \Gamma, \vec{n}\rangle=\left|C, \beta_{d}, \vec{q}, \Gamma_{d}, \vec{n}\right\rangle, \\
\hat{U}_{d}|C, \Gamma, \vec{n}\rangle=\left|C, \Gamma_{d}, \vec{n}\right\rangle .
\end{gathered}
$$

Here $\Gamma_{d}$ is the equivalence class of the 1 parameter set of graphs $\gamma_{d}(s)$, where $\gamma_{d}(s)$ is obtained by the action of $d$ on $\gamma(s)$ and the labelled graph $\beta_{d}, \vec{q}$ is the image of the labelled graph $\beta, \vec{q}$ by $d$. Note that if $\gamma(s)$ is obtained by the action of the diffeomorphism $\phi_{\xi}(s)$ on some graph $\gamma$ for some analytic vector field $\xi$, it follows that $\gamma_{d}(s)$ is obtained by the action of $\phi_{\xi_{d}}(s)$ on $\gamma_{d}$ where $\xi_{d}$ is the analytic vector field obtained by the action of $d$ on $\xi$ and $\gamma_{d}$ 
is the image of $\gamma$ by $d$. Thus, the right hand side of equation (51) is also in $\mathcal{B}$ and the assumption (11) is valid.

The reader may check explicitly that both the adjointness 9 and algebraic relations on these operators are represented through equations (31), (45), (46), (47), (48) and (51),(52).

\subsection{Open Issues and Remarks.}

\subsubsection{Infinitesmal and finite diffeomorphisms, $\mathcal{U}$ and $\mathcal{U D}$.}

Let the analytic vector field $\xi$ generate the one parameter family of diffeomorphisms $\phi_{\xi}(s)$. As shown below, the operators $\hat{U}_{\phi_{\xi}(s)}$ do not have the recquisite continuity properties in $s$ to define their generator as an operator on the dense domain $V_{\text {new }}$. Despite the inability to define such an operator on $V_{\text {new }}$, a more limited notion of infinitesmal diffeomorphisms does exist. Consider the state $|C, \Gamma, \vec{n}\rangle$ and let $\gamma(s)$ be obtained by dragging $\gamma$ along the orbits of the vector field $\xi$. As in section 3.2 , let $|C,\{\gamma(s), \vec{n}, s \in \mathbf{S}\}\rangle$ be in the equivalence class of $|C, \Gamma, \vec{n}\rangle$. Further, let $C(s)$ be smooth and let it be supported in $\mathbf{S}=\left[s_{1}, s_{2}\right]$ for some $s_{1}, s_{2} \in R$. From equation (52) it follows that for small enough $\delta$,

$$
\begin{array}{r}
\hat{U}_{\phi_{\xi}(s=\delta)}\left|C,\left\{\gamma(s), \vec{n}, s \in\left[s_{1}, s_{2}\right]\right\}\right\rangle=\left|C,\left\{\gamma(s+\delta), \vec{n}, s \in\left[s_{1}, s_{2}\right]\right\}\right\rangle \\
=\left|C_{\Delta},\left\{\gamma(s), \vec{n}, s \in\left[s_{1}+\delta, s_{2}+\delta\right]\right\}\right\rangle,
\end{array}
$$

where $C_{\Delta}(s):=C(s-\delta)$. Applying equation (8), we have that

$$
\begin{aligned}
\left(\hat{U}_{\phi_{\xi}(\delta)}-1\right)\left|C,\left\{\gamma(s), \vec{n}, s \in\left[s_{1}, s_{2}\right]\right\}\right\rangle & =\left|C_{\Delta}-C,\left\{\gamma(s), \vec{n}, s \in\left[s_{1}+\delta, s_{2}\right]\right\}\right\rangle \\
+\left|C_{\Delta},\left\{\gamma(s), \vec{n}, s \in\left[s_{2}, s_{2}+\delta\right]\right\}\right\rangle & -\left|C,\left\{\gamma(s), \vec{n}, s \in\left[s_{1}, s_{1}+\delta\right]\right\}\right\rangle \\
=\delta\left|C^{\prime},\left\{\gamma(s), \vec{n}, s \in\left[s_{1}, s_{2}\right]\right\}\right\rangle & +\delta^{2}|\psi\rangle,
\end{aligned}
$$

where $|\psi\rangle$ has norm of $O(1)$ as $\delta \rightarrow 0$ and where $C^{\prime}$ is a half density which, in the parametrization $s$, evaluates to $C^{\prime}(s):=\frac{\partial C(s)}{\partial s}$. Equation (54) implies that

$$
\lim _{\delta \rightarrow 0} \frac{\left(\hat{U}_{\phi_{\xi}(\delta)}-1\right)}{\delta}|C, \Gamma, \vec{n}\rangle=\left|C^{\prime}, \Gamma, \vec{n}\right\rangle .
$$

Equation (55) is the limited notion of infinitesmal diffeomorphisms which exists in the new representation. Note that for $\alpha$ such that $\alpha(s):=\phi_{\xi}(s) \alpha \neq$ $\alpha$ for all $s$ in some neighbourhood of the origin, the limit

$$
\lim _{\delta \rightarrow 0} \frac{\left(\hat{U}_{\phi_{\xi}(\delta)}-1\right)}{\delta}|C, \alpha, \vec{p}, \Gamma, \vec{n}\rangle
$$

\footnotetext{
${ }^{9}$ As mentioned in section $3.2, V_{\text {new }}$ is completed to the Hilbert space $\mathcal{H}_{\text {new }}$. Since $\hat{h}_{\alpha, \vec{p}}, \hat{U}_{d}$ are bounded operators, they admit unitary extensions to all of $\mathcal{H}_{\text {new }}$. However $\hat{E}_{\mathcal{S}, f}$ is unbounded and hence only densely defined with dense domain $V_{\text {new }}$. While it is straightforward to check that $\hat{E}_{\mathcal{S}, f}$ is symmetric on this domain, we do not address issues of self adjointness here other than to remark that it should be much easier to handle the bounded operators $\exp \left(i \hat{E}_{\mathcal{S}, f}\right)$.
} 
does not exist due to the fact that $\left\langle C, \alpha, \vec{p}, \Gamma, \vec{n}\left|\hat{U}_{\phi_{\xi}(\delta)}\right| C, \alpha, \vec{p}, \Gamma, \vec{n}\right\rangle=0$ for all small enough $\delta$. This shows that $\lim _{\delta \rightarrow 0} \frac{\left(\hat{U}_{\phi_{\xi}(\delta)}-1\right)}{\delta}$ does not exist on all of $V_{\text {new }}$.

We do not know if an operator for infinitesmal diffeomorphisms can be defined on some other dense domain in $\mathcal{H}_{\text {new }}$, but we consider it unlikely. We also feel that such an operator cannot be defined as a limit of operators in the holonomy flux algebra but this needs to be shown. Likewise, while we feel that the finite diffeomorphism operator $\hat{U}_{d}$ cannot be defined as a limit of operators in the holonomy- flux algebra, this too remains an open question. In the unlikely event that such an operator can be defined in this way, our intuition is that there must be at least one such definition which corresponds to the action of $\hat{U}_{d}$ as defined in equations (51)- (52). If our expectations our correct, the extension of $\mathcal{U}$ to $\mathcal{U D}$ and the subsequent analysis of this work is, we believe, fully justified.

\subsubsection{Cyclicity, GNS states and the flux operators.}

The new representation is not cyclic but cyclic subspaces can be defined via the standard Gelfand- Naimark- Segal (GNS) construction (see for example [10]). Any state $\Psi \in \mathcal{H}_{\text {new }}$ defines, via its expectation values, a positive linear functional (PLF), on $\mathcal{U D}$ (or any of its subalgebras). This PLF defines a cyclic representation (via the GNS construction) in which $\Psi$ is a cyclic state. Let $\Psi=|C, \Gamma, \vec{n}\rangle$ be a normalised state (so that $\int d s|C(s)|^{2}=1$ ) and consider the associated PLF evaluated on the commutative algebra of holonomies, $H A \subset \mathcal{U D}$. Clearly

$$
\begin{aligned}
\left\langle C, \Gamma, \vec{n}\left|\hat{h}_{\alpha, \vec{p}}\right| C, \Gamma, \vec{n}\right\rangle & =0 \forall \alpha \neq \circ \\
& =1 \text { for } \alpha=0 .
\end{aligned}
$$

This is exactly the standard PLF on $H A$ used to construct the flux net representation. The difference with the standard PLF arises when one considers the algebra $\mathcal{U}$ of holonomies and fluxes. By using the commutators (18)(19), any element of $\mathcal{U}$ can be expressed as a linear combination of terms, each of the form $\hat{h}_{\alpha, \vec{p}} \prod_{i=1}^{m} \hat{E}_{\mathcal{S}_{i}, f_{i}}$. From equations (47), (48) and (32), we have that

$$
\begin{aligned}
\left\langle C, \Gamma, \vec{n}\left|\hat{h}_{\alpha, \vec{p}} \prod_{i=1}^{m} \hat{E}_{\mathcal{S}_{i}, f_{i}}\right| C, \Gamma, \vec{n}\right\rangle & =0 \\
\left\langle C, \Gamma, \vec{n}\left|\prod_{i=1}^{m} \hat{E}_{\mathcal{S}_{i}, f_{i}}\right| C, \Gamma, \vec{n}\right\rangle & =\int d s|C(s)|^{2} \prod_{i=1}^{m} C_{\Gamma, \vec{n}}^{\mathcal{S}_{i}, f_{i}}
\end{aligned}
$$

In the standard flux network representation, any state in $\mathcal{H}$ is a linear combination of at most a countable infinity of flux network states. In contrast, 
equation (59) refers to the structure provided by the uncountably many graphs $\gamma(s)$.

Finally, the PLF evaluated on the remaining part of $\mathcal{U D}$ can be obtained by using equations (11)- (4) in conjunction with its evaluation on elements of the form $\hat{h}_{\alpha, \vec{p}}\left(\prod_{i=1}^{m} \hat{E}_{\mathcal{S}_{i}, f_{i}}\right) \hat{U}_{d}$. It is straightforward to evaluate this and we do not display the result here, other than to comment that the PLF vanishes unless $\alpha=0$.

\subsubsection{Reducibility.}

Denote the cyclic representation of $\mathcal{U D}$ considered above by $\left(\mathcal{U D}, \mathcal{H}_{C, \Gamma, \vec{n}}\right)$. Here $\mathcal{H}_{C, \Gamma, \vec{n}}$ is the GNS Hilbert space associated with the PLF of section 3.4.2. Irreducibility of $\left(\mathcal{U D}, \mathcal{H}_{C, \Gamma, \vec{n}}\right)$ is an involved issue and we do not address it in this work. However, it is straightforward to see that representation, $\left(\mathcal{U}, \mathcal{H}_{C, \Gamma, \vec{n}}^{\mathcal{U}}\right)$, obtained if we restrict the PLF of section 3.4.2 to the holonomy- flux algebra is, most likely, infinitely reducible. From equation (46), the operator $\hat{h}_{\alpha, \vec{p}}$ does not alter the half density label $C$ in $|C, \Gamma, \vec{n}\rangle$. However (see equation (48)) the flux operator $\hat{E}_{\mathcal{S}, f}$ does alter $C$ to $C_{1}:=C C_{\gamma, \vec{n}}^{\mathcal{S}, f}$. Clearly, by choosing $\mathcal{S}, f$ appropriately $C_{1}$ can have smaller support than $C$ but can never have larger support than $C$. Thus, the cyclic subspace generated by using the PLF defined by $\left|C_{1}, \Gamma, \vec{n}\right\rangle$ is invariant under the action of elements of $\mathcal{U}$. Repeated action by appropriately chosen flux operators yield further cyclic subspaces which are invariant with respect to $\mathcal{U}$ so that the GNS representation based on the PLF defined by $|C, \Gamma, \vec{n}\rangle$ and restricted to $\mathcal{U}$ is infinitely reducible with respect to $\mathcal{U}$. Note, however, that this argument pertains only to the cyclic subspace generated by the action of $\mathcal{U}$ on $|C, \Gamma, \vec{n}\rangle$. This subspace is dense in (and not equal to) the GNS Hilbert space $\mathcal{H}_{C, \Gamma, \vec{n}}^{\mathcal{U}}$. Hence, the argument as it stands, is not, strictly speaking, complete in that it does not adequately address issues of adjointness of the densely defined, unbounded flux operators. Thus, the notion of irreducibility in the context of $\left(\mathcal{U}, \mathcal{H}_{C, \Gamma, \vec{n}}^{\mathcal{U}}\right)$ is complicated by the fact that the flux operators are unbounded and hence only densely defined. However, it may be possible to show infinite reducibility in the context of $\mathcal{H}_{C, \Gamma, \vec{n}}^{\mathcal{U}}$ if (similar to the idea in Reference [11]), $\mathcal{U}$ is replaced by the algebra generated by holonomies and exponentials of electric fluxes (i.e. by the bounded, unitary operators $\left.e^{i \hat{E}_{\mathcal{S}, f}}\right)$.

This would be an undesirable feature if we has access only to $\mathcal{U}$ (or its Weyl algebra- like replacement alluded to above). However the operators $\hat{U}_{d}$ map states in $\mathcal{H}_{C, \Gamma, \vec{n}}^{\mathcal{U}}$ out of $\mathcal{H}_{C, \Gamma, \vec{n}}^{\mathcal{U}}$ and hence are not superselected with respect to $\mathcal{H}_{C, \Gamma, \vec{n}}^{\mathcal{U}}$. Moreover, it is conceivable that some of these operators (at least for some choices of $\Gamma$ ) may be used to "stretch" the support of $C$ by "stretching" the 1 parameter family of graphs $\gamma(s)$. It would be interesting to see if this is indeed possible, as it impinges on the issue of irreducibility 
of $\left(\mathcal{U D}, \mathcal{H}_{C, \Gamma, \vec{n}}\right)$.

\subsubsection{Inequivalence with the standard flux net representation.}

As noted in section 3.4.2, the flux operators have a very different action (based on a uncountable family of flux net labels) than in the standard representation (where it can depend at most on a countable infinity of flux net labels) and this difference shows their inequivalence. Yet another reason to believe that the representations are inequivalent can be traced to the nature of the eigen functions of the flux operators. In the standard representation, every flux net state is an eigen state of the flux operators and every such state is normalizable. In the new representation, equations (47)- (48) suggest that eigen states may be obtained by replacing $C(s)$ in those equations by the Dirac delta function $\delta\left(s_{0}, s\right)$, where $\delta\left(s_{0}, s\right)$ is a half density in each of its arguments. Clearly, such a replacement yields a nonnormalizable state (also see Footnote 3 in this regard). A third way to demonstrate inequivalence would be to show that no diffeomorphism invariant state exists in $\mathcal{H}_{\text {new }}$. Direct inspection shows that there is no such state in $V_{\text {new }}$. Since any state $|C, \alpha, \vec{p}, \Gamma, \vec{n}\rangle$ is associated with a graph and a finite number of analytic surfaces traced out by the edges of $\gamma(s)$ (see Lemma 1 in the Appendix), it follows that any state in $\mathcal{H}_{\text {new }}$ is associated with, at most, a countable infinity of graphs and surfaces. Since the number of (finite) analytic diffeomorphisms is uncountably large, it seems unlikely that a diffeomorphism invariant state exists in $\mathcal{H}_{\text {new }}$. It would be of interest to convert these arguments into rigorous proofs.

As noted in section 3.4.2 the PLF based on $|C, \Gamma, \vec{n}\rangle$ restricted to the holonomy algebra $H A$ is identical to the standard PLF appropriate to the flux net representation. Hence there seems to be enough structure to define the space $\overline{\mathcal{A}}$ of generalised connections [12]. The flux operators in the standard representation are related to derivations on this space; it would be of interest to see if the flux operators in the new representation have any interpretation in terms of structures on $\overline{\mathcal{A}}$. This issue has a bearing on the discussion centered around (1) in section 1.

\section{Concluding Remarks.}

In this work we have constructed a new representation for a diffeomorphism invariant theory of abelian connections. In the new representation, finite diffeomorphisms act unitarily. It is in this sense that the representation is "background independent". From the point of view of quantum states as positive linear functionals, the key difference between the standard flux net representation and the one constructed here is in the evaluation of the PLF on electric flux operators (59). There are a number of open questions regarding the new representation and we urge the reader to peruse section 
3.4 wherein they are described in detail. We emphasise once again that though the cyclic sector (see section 3.4.2) of the new representation is, most likely, infinitely reducible when defined with respect to the holonomyflux algebra $\mathcal{U}$ this is probably not the case with respect to the holonomyflux- diffeomorphism algebra $\mathcal{U D} .10$ Moreover, if our intuition is correct, the operators $\hat{U}_{d}$ corresponding to finite diffeomorphisms either cannot be defined as limits of operators in $\mathcal{U}$ or can be defined in terms of such limits in such a way as to agree with their definition in the new representation. If this is true, then there is no reason not to take the algebra $\mathcal{U D}$ as a starting point for quantization rather than the algebra $\mathcal{U}$.

In this work we have restricted attention to the analytic category. However we expect our considerations to be robust enough to generalise easily to the semianalytic category as well.

We leave a generalization of our constructions to the case of gauge group $S U(2)$ for future work. Note that there may be implementations of the ideas of section 2 which are different from the sort of constructions in section 3 . For example, one could attempt to define a 1 parameter family of states in the non- abelian case by first considering a (non gauge invariant) spinnet based on a loop with a single analytic edge, a single vertex with an intertwiner and a vector in an appropriate representation of the gauge group [8], and then generating the 1 parameter family by moving the vertex and its interwtwiner and vector labels along the loop - the parameter in this case is the position of the vertex. It would be of interest to see if this (or other ideas) can lead to interesting new representations. Finally, we hope that the work here, in particular the form of the GNS functional (57), (59), may motivate the constructions of alternate representations for canonical quantum gravity.

Acknowledgments: This work could not have been completed without Hanno Sahlmann's help. I gratefully acknowledge crucial discussions with him and thank him for his constant encouragement. I also gratefully acknowledge an invitation from T. R. Seshadri to visit the Physics Department of Delhi University where part of this work was completed.

\section{Appendix}

Lemma 1 Let $\Sigma$ be a real analytic, 3 dimensional, compact manifold (without boundary). Let $e$ be a non- self intersecting analytic curve in $\Sigma$ i.e. $e: T \rightarrow \Sigma$ is an injective analytic map from an open interval $T$ of the real line $R$ into $\Sigma$. Let $\xi$ be an analytic vector field on $\Sigma$ and $U \subset \Sigma$ an open set such that $\xi$ is non- vanishing in $U$ and $e(t) \subset U \forall t \in T$. Let $\phi_{\xi}(s), s \in R$,

\footnotetext{
${ }^{10}$ As indicated in section 3.4.2 a precise definition of irreducibility is only available for algebras of bounded operators and hence, strictly speaking, the flux operators in $\mathcal{U}, \mathcal{U D}$ should be replaced by suitable bounded functions thereof (see section 3.4.2 for further discussion of this).
} 
denote the one parameter family of diffeomorphisms of $\Sigma$ generated by $\xi$ with $\phi_{\xi}(0)$ being the identity map. Let there exist an open neighbourhood, $S$, of the origin in $R$ such that $e(s, t):=\phi_{\xi}(s) e(t) \subset U \forall s \in S, t \in T$. Further, let the image, $\phi_{\xi}(s) e$ of the edge $e$ under the diffeomorphism $\phi_{\xi}(s)$ be transverse to $\xi \forall s \in S$.

Then the set

$$
S_{\xi, S, T}=\{e(t, s) \forall s \in S, t \in T\}
$$

is an analytic 2- surface in $\Sigma$ with analytic chart $(s, t)$.

Proof: Standard results for the smooth category imply that $S_{\xi, S, T}$ is a smooth 2- surface with chart $(s, t)$. Clearly, given $\left(t_{0}, s_{0}\right) \in T \times S$, there exist open neighbourhoods $V_{S} \subset S, V_{T} \subset T$ and $V \subset \Sigma$ such that:

(a) $\left(t_{0}, s_{0}\right) \in V_{T} \times V_{S}$,

(b) $V$ is covered by a single analytic chart,

(c) $\left\{e(t, s) \forall(t, s) \in V_{T} \times V_{S}\right\} \subset V$

Since $\phi_{\xi}(s)$ is a 1 parameter family of analytic diffeomorphisms 11 and $e(t)$ is an analytic curve, it follows that $e^{\mu}\left(t, s_{0}\right), t \in V_{T}, \mu=1,2,3$ are analytic functions of $t$ (here $e^{\mu}(t, s)$ are the coordinates of $(e(t, s)$ in the analytic chart on $V$ ).

The surface $S_{\xi, S, T}$ is defined by the "evolution" equations

$$
\frac{\partial e^{\mu}}{\partial s}=\xi^{\mu}(e(t, s))
$$

and the initial conditions

$$
e^{\mu}(t, 0)=e^{\mu}(t)
$$

Our strategy is to show that equations (61) with initial data (62) admit unique analytic solutions. This follows directly from an application of the Cauchy- Kowalewsky theorem [9]. In order to apply the theorem in the form specified in Reference [9, we proceed as follows.

We set $e^{\mu}\left(t_{0}, s_{0}\right)=0$. 12 Define the new coordinates $t^{\prime}:=t-t_{0}$ on $U_{T}$ and $s^{\prime}=s-s_{0}$ on $U_{S}$. Define

$$
\begin{gathered}
u_{i}=e^{i}\left(t^{\prime}, s^{\prime}\right):=e^{i}(t, s), \quad i=1,2,3 . \\
u_{4}:=t^{\prime}, x^{1}:=t^{\prime} .
\end{gathered}
$$

\footnotetext{
${ }^{11}$ While it seems to be standard folklore that analytic vector fields generate analytic diffeomorphisms, we are unable to locate this result in the literature. However, we have verified that (in the context of compact manifolds without boundary, where results for the smooth category hold) an application of the Cauchy- Kowalewsky theorem [9] proves the result.

${ }^{12}$ That this entails no loss of generality follows from the fact that translation by a constant is an analytic transformation.
} 
Define the functions $F_{i, j, k}\left(u_{1}, u_{2}, u_{3}, u_{4}\right), i, j=1, . ., 4, k=1$ as follows

$$
\begin{aligned}
& F_{i, j, 1}=0 \text { for } i, j=1,2,3 . \\
& F_{i, 4,1}=\xi^{i}\left(u_{1}, u_{2}, u_{3}\right) i=1,2,3 . \\
& F_{4, i, 1}=0 \text { for } i=1,2,3,4 .
\end{aligned}
$$

It is straightforward to check that the content of the evolution equations (61) with initial data (62) can now be re-expressed in the evolution equations

$$
\frac{\partial u_{i}}{\partial s^{\prime}}=\sum_{j=1}^{4} F_{i, j, k} \frac{\partial u_{j}}{\partial x^{k}} \quad i=1,2,3,4 \quad k=1
$$

with initial data

$$
\begin{gathered}
u_{i}\left(t^{\prime}, 0\right)=e^{i}\left(t^{\prime}, 0\right)=e^{i}\left(t, s_{0}\right) \quad i=1,2,3 \\
u_{4}\left(t^{\prime}, 0\right)=t^{\prime} .
\end{gathered}
$$

The analyticity of $F_{i, j, k}$ in the neighbourhood of the origin in $R^{4}$ follows from the analyticity of $\xi^{\mu}$; the analyticity of the initial data (69) follows from the analyticity of the curve $e\left(t, s_{0}\right)$ (and the fact that the coordinates $t$ and $t^{\prime}$ as well as $s$ and $s^{\prime}$ are analytic functions of each other) whereas the initial data (70) is trivially analytic. Finally, it can be checked that the initial data (69) - (70) vanish at the origin $\left(t^{\prime}, s^{\prime}\right)=(0,0)$. Thus all the conditions of Theorem 2.4.1 of [9] are met and the equations (68) - (70) are identical to equations (2.43) and (2.44) of [9]. It follows that there exist solutions $u_{i}\left(t^{\prime}, s^{\prime}\right), i=1, . ., 4$ in a neighbourhood of the origin $\left(t^{\prime}, s^{\prime}\right)=(0,0)$ which are analytic functions of $\left(t^{\prime}, s^{\prime}\right)$. Since $t=t^{\prime}+t_{0}, s=s^{\prime}+s_{0}$ are analytic functions of $t, s$, it follows that there exists an open neighbourhood of $\left(t=t_{0}, s=s_{0}\right)$ where $e^{\mu}(s, t), \mu=1,2,3$ are analytic functions of $(s, t)$. Since $\left(t_{0}, s_{0}\right)$ is an arbitrary point on $S_{\xi, S, T}$, this completes the proof of the Lemma.

\section{References}

[1] C. Fleischhack eprint math-ph/0407006; C. Fleischhack, Phys.Rev.Lett.97, 061302 (2006); A. Okolow and J. Lewandowski, Class.Quant.Grav.20, 3543 (2003); A. Okolow and J. Lewandowski, Class.Quant.Grav.22, 657 (2005). H. Sahlmann, e-print gr-qc/0207112 and eprint gr-qc/0207111; H. Sahlman and T. Thiemann, eprint gr-qc/0302090 H. Sahlmann and T. Thiemann, Class.Quant.Grav.23, 4453 (2006).

[2] C. Fleischhack in Quantum Gravity: Mathematical Models and Experimental Bounds edited by Fauser et. al., Birkhauser Basel, 2007. 
[3] J. Lewandowski, A. Okolow, H. Sahlmann and T. Thiemann, Comm.Math.Phys.267, 703 (2006).

[4] A. Ashtekar, A. Corichi and J. A. Zapata, Class.Quant.Grav.15, 2955 (1998).

[5] M. Varadarajan, Phys.Rev.D64, 104003 (2001).

[6] A. Ashtekar and J. Lewandowski, Class.Quant.Grav.18, L117 (2001).

[7] A. Ashtekar and J. Lewandowski, Class.Quant.Grav.21, R53 (2004).

[8] A. Ashtekar and J. Lewandowski,Class.Quant.Grav.14, A55 (1997).

[9] Theorem 2.4.1 of A primer of Analytic Functions, S. G. Krantz and H. R. Parks, (Second Edition, Birkhauser).

[10] See for example Quantum Field Theory in Curved Spacetime and Black Hole Thermodynamics, R. W. Wald (The University of Chicago Press 1994).

[11] H. Sahlmann and T. Thiemann, Class.Quant.Grav.23, 4453 (2006).

[12] A. Ashtekar and J. Lewandowski, J.Math.Phys.36, 2170 (1995). 